\section{Should a Course of Prophylactic Probiotics Accompany Inpatient Antibiotic Treatment for Adults?}

To the Editor: Hospital censuses are at historic highs due to Coronavirus disease 2019 (COVID-19). As a result, we are experiencing an increased length of inpatient stays and prolonged treatments with antibiotics and steroids in our elderly patients who suffer the most from this illness.

Clostridioides difficile (" $C$ diff") colitis is the most common hospital-acquired infection that also has the risk factors above. It also shares a presenting symptom with COVID-19 (diarrhea) and delays recognition of comorbid disease, leading to further morbidity and mortality. Nearly every class of antibiotics, including $C$ diff treatments, is associated with $C$ diff infection development. ${ }^{1}$

$C$ diff infection is a significant burden to the patient and health care system, with a $9.3 \%$ 30-day mortality rate $^{2}$ and annual costs estimated at $\$ 1.5$ billion in 2009. ${ }^{3}$ In 2011, in the United States, $C$ diff attributed to 29,000 deaths alone. ${ }^{2}$

A systematic review by Goldenberg et al, ${ }^{4}$ suggested probiotic use versus placebo reduces $\mathrm{C}$ diff infection incidence by $60 \%$, with a number-needed-to-treat (NNT) of 42 to prevent an infection. Rates of adverse events were not significantly different from placebo. The typical intervention used across the component studies is easy to perform in the hospital: 1 probiotic twice daily for 2 to 3 weeks or the duration of antibiotic treatment.

Notably, patients on chemotherapy were excluded from the above trials; however, a Cochrane review found no severe adverse effects associated with their use to prevent or treat chemotherapy-associated diarrhea. ${ }^{5}$ One should also not discount other benefits of probiotics, such as preventing general antibiotic-associated diarrhea (NNT of 17). ${ }^{4}$

A recent systematic review addressed the cost-effectiveness of prophylactic probiotics, with all but 1 study demonstrating the benefit of probiotic use in conjunction with antibiotics for preventing complications and hospital-acquired infections. Estimated potential savings of $\$ 500$ to $\$ 2700$ per patient versus placebo were reported. ${ }^{6}$ The included studies are limited by low-quality evidence.

Lack of standardization and heterogeneity of treatment remain challenges to implementing probiotic therapies in clinical practice. However, the positive results and favorable safety profiles across studies are reassuring. The regimens with the strongest evidence include $S$ boulardii; a 2-strain combination of $L$ acidophilus CL1285 and Lactobacillus casei LBC80R; a 3-strain combination of $L$ acidophilus, Lactobacillus delbrueckii subsp bulgaricus, and
Bifidobacterium bifidum; or the 4-strain combination of $L$ acidophilus, $L$ delbrueckii subsp bulgaricus, B bifidum, and Streptococcus salivarius subsp thermophilus.

Our best method of preventing $C$ diff infections is multifactorial - a combination of judicious antibiotic use and effective hygiene/sanitization. Based on compelling evidence, we argue for probiotic use, especially for the elderly hospitalized patient on antibiotics. Probiotics as prophylaxis for outpatient treatment in elderly populations is worth considering as well, given the safety profile and potential for preventing a potentially devastating complication.

Vijay Venkatesan, MD Atrium Health Carolinas Medical Center, Family Medicine Residency Program, 2001 Vail Ave Ste 400,

Charlotte, NC 28207 vkvenkatesan@outlook.com

Jay Indravadan Patel, MD

To see this article online, please go to: bttp://jabfm.org/content/ 34/5/1017.full.

\section{References}

1. Leffler DA, Lamont JT. Clostridium difficile infection. N Engl J Med 2015;372:1539-48.

2. Lessa FC, Mu Y, Bamberg WM, et al. Burden of Clostridium difficile infection in the United States. N Engl J Med 2015;372:825-34.

3. Zimlichman E, Henderson D, Tamir O, et al. Health care-associated infections: a meta-analysis of costs and financial impact on the US health care system. JAMA Intern Med 2013;173:2039-46.

4. Goldenberg JZ, Yap C, Lytvyn L, et al. Probiotics for the prevention of Clostridium difficile-associated diarrhea in adults and children. Cochrane Database Syst Rev 2017; 12(12):CD006095. May 31.

5. Wei D, Heus P, van de Wetering FT, van Tienhoven G, Verleye L, Scholten RJ. Probiotics for the prevention or treatment of chemotherapy- or radiotherapy-related diarrhoea in people with cancer. Cochrane Database Syst Rev 2018;8:CD008831.

6. Lau VI, Rochwerg B, Xie F, et al. Probiotics in hospitalized adult patients: a systematic review of economic evaluations. Can J Anaesth 2020;67:247-61.

7. Su GL, Ko CW, Bercik P, et al. AGA clinical practice guidelines on the role of probiotics in the management of gastrointestinal disorders. Gastroenterol 2020;159:697-705.

doi: 10.3122/jabfm.2021.05.210158 